\title{
DIFFERENTIABILITY OF THE METRIC PROJECTION IN HILBERT SPACE
}

BY

\author{
SIMON FITZPATRICK AND R. R. PHELPS ${ }^{1}$
}

\begin{abstract}
A study is made of differentiability of the metric projection $P$ onto a closed convex subset $K$ of a Hilbert space $H$. When $K$ has nonempty interior, the Gateaux or Fréchet smoothness of its boundary can be related with some precision to Gateaux or Fréchet differentiability properties of $P$. For instance, combining results in $\$ 3$ with earlier work of R. D. Holmes shows that $K$ has a $C^{2}$ boundary if and only if $P$ is $C^{1}$ in $H \backslash K$ and its derivative $P^{\prime}$ has a certain invertibility property at each point. An example in $\$ 5$ shows that if the $C^{2}$ condition is relaxed even slightly then $P$ can be nondifferentiable (Fréchet) in $H \backslash K$.
\end{abstract}

1. Introduction. Suppose that $H$ is a real Hilbert space and that $K$ is a nonempty closed convex subset of $H$. For each $x \in H$ we let $P_{K} x$ (or simply $P x$ ) denote the unique element of $K$ which satisfies

$$
\|x-P x\|=\inf \{\|x-y\|: y \in K\} .
$$

This well-known mapping, called either the nearest-point mapping or the metric projection of $H$ onto $K$, is easily seen to be nonexpansive, that is,

$$
\|P x-P y\| \leqslant\|x-y\|, \quad x, y \in H .
$$

This Lipschitzian property guarantees, for instance, that $P_{K}$ is Gateaux differentiable "almost everywhere" when $H$ is separable (see, for instance, [7] and references cited therein), and several authors (Zarantonello, [10] Haraux [3] and Mignot [6]) have shown the existence of directional derivatives for $P_{K}$ at the boundary of $K$, the latter two having applied this fact to certain variational inequalities. In this paper we will study both the Gateaux and Fréchet derivatives of $P_{K}$, examining the question of their existence and of their relationship to the local geometric structure of $K$. As might be expected, there is a close connection between the existence of the Fréchet derivative and the smoothness of the boundary of $K$; by theorem and counterexample we are able to delineate this connection rather precisely.

After writing the initial draft of this paper we became aware of Holmes' work [4] which anticipated one of our smoothness results: If $K$ has a $C^{k+1}$ boundary, then $P_{K}$ is $C^{k}$ in $H \backslash K$, and its Fréchet derivative has a certain invertibility property at each point. We prove the converse to this result and, by means of a rather surprising

Received by the editors April 14, 1980.

1980 Mathematics Subject Classification. Primary 41A50, 41A65, 46C05, 47H99.

Key words and phrases. Gateaux derivative, Fréchet derivative, metric projection, nearest-point map, convex body, Hilbert space, Minkowski functional, gauge functional.

${ }^{1}$ Research supported in part by a grant from the National Science Foundation. 
example, we show that there exists a set $K$ with $C^{2-}$ boundary such that $P_{K}$ is nowhere Fréchet differentiable in $H \backslash K$. We also examine various questions concerning the existence of the Gateaux or Fréchet derivative of $P_{K}$ at a single point, and we characterize $C^{1}$ smoothness of the boundary of $K$ in terms of a certain partial differentiability property of $P_{K}$.

For an extraordinarily thorough study of metric projections we direct the reader to Zarantonello's book-length paper [10]; much of what we have done was motivated by his work.

The definition of $P_{K}$ is equivalent, of course, to the condition that for each $x$ in $H$ $\left\|x-P_{K} x\right\| \leqslant\|x-y\| \quad$ whenever $y \in K$.

It is elementary [10] to show that this is in turn equivalent to

$$
\left\langle x-P_{K} x, P_{K} x-y\right\rangle \geqslant 0 \text { for each } y \in K .
$$

The latter inequality (which will be referred to as the defining inequality for $P_{K}$ ) has a simple geometric interpretation: The linear functional on $H$ defined by the inner product with the vector $x-P_{K} x$ attains its maximum on $K$ at the point $P_{K} x$. Another way of looking at this is to define, for $x \in H \backslash K$,

$$
H[x]=\left\{y \in H:\left\langle x-P_{K} x, y\right\rangle=0\right\},
$$

the hyperplane orthogonal to the (nonzero) vector $x-P_{K} x$; one then notes that the translate $H[x]+P_{K} x$ supports the convex set $K$ at $P_{K} x$.

Let $E, F$ be real Banach spaces, $U$ a nonempty open subset of $E$ and $f: U \rightarrow F$ a continuous function. We say that $f$ is Gateaux differentiable at a point $x \in U$ if

$$
\lim _{t \rightarrow 0} \frac{f(x+t h)-f(x)}{t}
$$

exists for each $h \in E$ and is linear and continuous in $h$. This linear operator, when it exists, is denoted by $d f(x)$. If the above limit exists uniformly for $h$ in the unit ball of $E$ (and is linear and continuous in $h$ ) we say that $f$ is Fréchet differentiable at $x$ and denote the corresponding linear operator by $f^{\prime}(x)$. These two definitions can be restated in the following form:

The operator $d f(x)$ is characterized by $f(x+t h)=f(x)+t d f(x) h+o(t)(h \in E)$ where $o(t) /|t| \rightarrow 0$ as $t \rightarrow 0$.

The operator $f^{\prime}(x)$ is characterized by $f(x+y)=f(x)+f^{\prime}(x) y+o(y)$, where $o(y) /\|y\| \rightarrow 0$ as $y \rightarrow 0$.

2. Basic properties of the derivatives of $P_{K}$. When they exist, the bounded linear operators $d P(x)$ and $P^{\prime}(x)$ have some elementary operator-theoretic properties (such as symmetry and positivity) which do not depend on $K$; this section will be devoted to such results. Since $d P(x)=P^{\prime}(x)$ when the latter exists, our proofs can be restricted to the Gateaux derivative. We will usually assume that $x \in H \backslash K$; this is no real loss in generality, since (as will be seen in §3) the derivative of $P_{K}$ exists for points of $K$ only in trivial cases.

2.1 Proposition. The operators $P^{\prime}(x)$ and $d P(x)$ have norm at most 1 , and hence $I-P^{\prime}(x)$ and $I-d P(x)$ are positive operators, that is, $\langle h-d P(x) h, h\rangle \geqslant 0$ for all $h$ in $H$. 
Proof. For $t \neq 0$ and any $h \in H$ we have

$$
\begin{aligned}
\|d P(x) h\| & \leqslant|t|^{-1}(\|P(x+t h)-P x\|+\|o(t)\|) \\
& \leqslant\|h\|+|t|^{-1}\|o(t)\|,
\end{aligned}
$$

the latter inequality holding because $P$ is nonexpansive, so $d P(x)$ clearly has norm at most 1 . To see the positivity assertion, note that for any $h$ in $H$,

$$
\langle h, d P(x) h\rangle \leqslant\|h\| \cdot\|d P(x) h\| \leqslant\|h\|^{2}=\langle h, h\rangle,
$$

so $0 \leqslant\langle h, h-d P(x) h\rangle$.

The first assertion in the next result is implicit in Asplund's work [1] and essentially the same result has been proved by Zarantonello [11] and Holmes [4]. For the sake of completeness we sketch a proof.

2.2 Proposition. The metric projection $P$ onto the closed convex set $K$ is the Fréchet derivative of the (convex) function

$$
f(x)=\frac{1}{2}\|x\|^{2}-\frac{1}{2}\|x-P x\|^{2}, \quad x \in H .
$$

Consequently, when $P^{\prime}(x)$ (or $\left.d P(x)\right)$ exists it is the second derivative of $f$, hence is a positive and symmetric operator; that is, we always have $\langle u, d P(x) u\rangle \geqslant 0$ and

$$
\langle u, d P(x) v\rangle=\langle d P(x) u, v\rangle \quad(u, v \in H) .
$$

Proof. It suffices to show that for fixed $x$ and any $h \in H$, we have

$$
f(x+h)-f(x)-\langle P x, h\rangle=o(h) .
$$

First, since $\|(x+h)-P(x+h)\| \leqslant\|x+h-P x\|$, we have $f(x+h)-f(x)-$ $\langle P x, h\rangle \geqslant 0$. On the other hand, since $\|x-P x\| \leqslant\|x-P(x+h)\|$ we get

$$
\begin{aligned}
f(x+h)-f(x)-\langle P x, h\rangle & \leqslant\langle h, P(x+h)-P x\rangle \\
& \leqslant\|h\| \cdot\|P(x+h)-P x\| \leqslant\|h\|^{2}=o(h) .
\end{aligned}
$$

The proof in [2] that $f^{\prime \prime}(x)$ is always symmetric utilizes the Fréchet derivative, but takes place entirely in the finite dimensional subspace generated by $x, u$ and $v$, hence applies equally well to the Gateaux derivative of $f^{\prime}=P$. The convexity of $f$ can be verified directly or one can write

$$
2 f(x)=\|x\|^{2}-\inf \left\{\|x-y\|^{2}: y \in K\right\}=\sup \left\{-2\langle x, y\rangle+\|y\|^{2}: y \in K\right\}
$$

and recall that a supremum of affine functions is convex. Finally, the second derivative of a convex function is a positive operator [8].

The next proposition shows that $d P(x)$ and $P^{\prime}(x)$ are (when they exist) within an orthogonal projection of being operators in the hyperplane $H[x]$.

2.3 Proposition. If $x \in H \backslash K$ let $P_{x}: H \rightarrow H[x]$ denote the orthogonal projection of $H$ onto $H[x]$. Then

$$
d P(x) \circ P_{x}=d P(x)=P_{x} \circ d P(x)
$$

in particular, $d P(x)$ maps $H[x]$ into itself. The same identity is valid for $P^{\prime}(x)$ when it exists. 
Proof. The first equality will follow from $d P(x)\left(y-P_{x} y\right)=0(y \in H)$. Since $y-P_{x} y \in H[x]^{\perp}=R(x-P x)$, it suffices to prove that $d P(x)(x-P x)=0$. But this is immediate from the definition of the Gateaux derivative and the fact that $P[x+t(x-P x)]-P x=0$ whenever $t>0$. The second equality is a consequence of the fact that since $P_{x}$ and $d P(x)$ are symmetric, they necessarily commute.

2.4 Corollary. If $x \in H \backslash K$ and $d P(x)$ (or $P^{\prime}(x)$ ) exists and maps $H$ onto $H[x]$, then its restriction to $H[x]$ is invertible.

Proof. If $d P(x)$ maps $H$ onto $H[x]$, then (by Proposition 2.3) its restriction to $H[x]$ is surjective; we now use the fact that a surjective and symmetric bounded operator is bijective hence, by the open mapping theorem, invertible.

2.5 Proposition. Suppose that $x \in H \backslash K$. If $d P(x)\left[P^{\prime}(x)\right]$ exists, then $d P(y)$ $\left[P^{\prime}(y)\right]$ exists for each point $y$ of the form

$$
y=\lambda x+(1-\lambda) P x, \quad \lambda>0,
$$

that is, for each point $y$ on the ray from Px through $x$. If $d P(x)$ is one-one (or surjective) in $H[x]$, then $d P(y)$ is one-one (or surjective) in $H[y]=H[x]$. Moreover, if $P$ is $C^{k}$ in a neighborhood of $x$, then it is $C^{k}$ in a neighborhood of $y$.

Proof. Since $P y=P(\lambda x+(1-\lambda) P x)=P x$ it is easy to see what form $d P(y)$ or $P^{\prime}(y)$ must have; in the latter case, for instance, the chain rule yields

$$
P^{\prime}(y) \circ\left[\lambda I+I(1-\lambda) P^{\prime}(x)\right]=P^{\prime}(x)
$$

hence $P^{\prime}(y)=P^{\prime}(x) \circ\left[\lambda I+(1-\lambda) P^{\prime}(x)\right]^{-1}$. To see that this formula is, in fact, correct, we carry out the proof for the Gateaux derivative; the same kind of argument serves for the Fréchet derivative.

Note first that $\lambda I+(1-\lambda) d P(x)$ actually is invertible. To show this we use Propositions 2.1 and 2.2 and the fact that an operator of the form $I+T$ is invertible whenever $T$ is positive. First, if $0<\lambda \leqslant 1$ then we can write

$$
\lambda I+(1-\lambda) d P(x)=\lambda\left[I+\lambda^{-1}(1-\lambda) d P(x)\right],
$$

while if $\lambda>1$, then

$$
\lambda I+(1-\lambda) d P(x)=I+(\lambda-1)[I-d P(x)]
$$

in either case we get essentially the identity operator plus a positive operator. Next, write $A$ in place of the invertible operator $\lambda I+(1-\lambda) d P(x)$; we want to show that for any $v \in H$

$$
P(y+t v)=P y+t d P(x)\left(A^{-1} v\right)+o(t) .
$$

This is equivalent (after writing $u=A^{-1} v$ ) to

$$
P(y+t A u)=P y+t d P(x) u+o(t) .
$$

Now,

$$
y+t A u=\lambda(x+t u)+(1-\lambda)(P x+t d P(x) u)
$$

and

$$
P(x+t u)=P x+t d P(x) u+o(t)
$$


so

$$
y+t A u=\lambda(x+t u)+(1-\lambda) P(x+t u)+o(t) .
$$

Since $P$ is nonexpansive and satisfies $P[\lambda(x+t u)+(1-\lambda) P(x+t u)]=$ $P(x+t u)$, this yields $P(y+t A u)=P(x+t u)+o(t)=P y+t d P(x) u+o(t)$, which was to be shown.

To prove the next assertion, note that we have shown that $d P(y)=d P(x) \circ A^{-1}$. It follows easily from this that the restriction of $d P(y)$ to the hyperplane $H[y]=$ $H[x]$ is one-one or onto if and only if $d P(x)$ has the same behavior. Indeed, this is immediate once it is observed that $A(H[x]) \subseteq H[x]$, that is, once we note that $\langle z, x-P x\rangle=0$ implies (using Proposition 2.3)

$$
\langle A z, x-P x\rangle=\lambda\langle z, x-P x\rangle+(1-\lambda)\langle d P(x) z, x-P x\rangle=0 .
$$

Suppose, finally, that $P$ is (Fréchet) $C^{k}$ in a neighborhood of $x$. Consider the function $F u=(\lambda I+(1-\lambda) P) u$; this is $C^{k}$ in a neighborhood of $x$ and since its derivative $F^{\prime}(x)=\lambda I+(1-\lambda) P^{\prime}(x)$ at $x$ is (as we have shown above) invertible, by the inverse function theorem there exist open neighborhoods $U$ of $x$ and $V$ of $y=F x$ and a $C^{k}$ function $G: V \rightarrow U$ such that $F(G v)=v$ for $v \in V$. Since $P(F u)=P u$ for all $u \in H \backslash K$ we have, for $v \in V, P(v)=P[F(G v)]=P(G v)$; this shows that $P$ is $C^{k}$ in $V$.

3. The relationship between smoothness of $K$ and smoothness of $P_{K}$. Throughout most of this section we will be working with a closed convex set $K$ that has nonempty interior, and we will characterize the smoothness of the boundary of $K$ in terms of differentiability properties of $P_{K}$. The simplest and most useful definition of " $C^{k}$ boundary" for this purpose is in terms of the Minkowski or (gauge) functional $\mu$ for $K$. Recall that if $x_{0} \in$ int $K$, then the Minkowski functional $\mu$ for $K$ with respect to $x_{0}$ is defined by

$$
\mu(x)=\inf \left\{t>0: x \in t\left(K-x_{0}\right)+x_{0}\right\}, \quad x \in H .
$$

This is a nonnegative convex continuous function which vanishes at $x_{0}$ (and perhaps elsewhere) and satisfies $\mu\left[r\left(x-x_{0}\right)+x_{0}\right]=r \mu(x)$ whenever $x \in H, r>0$. (In particular, $\mu$ is positive homogeneous if $x_{0}=0$.) Of special interest to us is the fact that the boundary $\partial K$ of $K$ is described by $\partial K=\{x \in H: \mu(x)=1\}$.

Definition. We say that the boundary $\partial K$ of $K$ is $C^{k}(k \geqslant 1)$ if for some $x_{0} \in$ int $K$ the Minkowski functional $\mu$ with respect to $x_{0}$ is $k$ times continuously Fréchet differentiable in some neighborhood of $\partial K$.

It is not difficult to apply the implicit function theorem to show that this definition is independent of the choice of the point $x_{0}$. Holmes [4] has shown that it is equivalent to $\partial K$ being (locally) a $C^{k}$ embedding in $H$ of an open subset of a hyperplane in $H$; this also shows that the definition is independent of the choice of $x_{0}$. In view of these remarks we lose no generality in assuming that $0 \in$ int $K$ and in working with the Minkowski functional for $K$ with respect to the origin. Henceforth, we will use $\mu$ to denote this Minkowski functional.

In discussing the smoothness of the boundary of $K$ (or of a convex continuous function) it is worthwhile to keep in mind that if a convex continuous function $f$ is 
Fréchet differentiable on some open convex set $U$, then it is in fact $C^{1}$ in $U$. If $f$ is Gateaux differentiable in $U$, then the differential map $x \rightarrow d f(x)$ is continuous from the norm to the weak topology. If it is norm to norm continuous, then $f$ is actually Fréchet differentiable.

We next collect some basic facts about the derivatives of a Minkowski functional.

3.1 Proposition. Suppose that $0 \in$ int $K$ and that $\mu$ is the Minkowski functional for $K$. Then

(i) If $\mu(x)>0$ and if $d \mu(x)$ exists, then $y=\mu(x)^{-1} x \in \partial K, y+d \mu(x) \notin K$ and $P(y+d \mu(x))=y$.

(ii) If $x \in H \backslash K$ and $d \mu(P x)$ exists, then it equals $\langle P x, x-P x\rangle^{-1}(x-P x)$.

(iii) If $d \mu(x)$ exists and $r>0$, then $d \mu(r x)$ exists and equals $d \mu(x)$.

(iv) If $d \mu$ exists in a neighborhood of $x$ and if the second derivative $d^{2} \mu$ exists at $x$, then $d^{2} \mu(x) x=0$.

Whenever $\mu^{\prime}$ exists it equals $d \mu$, so the assertions above are valid for the Fréchet derivative.

Proof. Part (iii) follows directly from the positive homogeneity of $\mu$ and (iv) follows from (iii). To prove (i), note that $\mu(y)=1$, so $y \in \partial K$. The vector $d \mu(x)$ is a subdifferential of $\mu$, that is,

$$
\langle d \mu(x), u-x\rangle \leqslant \mu(u)-\mu(x), \quad u \in H
$$

equivalently,

$$
\langle d \mu(x), u\rangle \leqslant \mu(u), \quad u \in H, \text { and }\langle d \mu(x), x\rangle=\mu(x) .
$$

In particular

$$
\langle d \mu(x), u\rangle \leqslant 1, \quad u \in K, \quad \text { and }\langle d \mu(x), y\rangle=1 .
$$

Consequently, $\langle d \mu(x), y+d \mu(x)\rangle=1+\|d \mu(x)\|^{2}>1$, so $y+d \mu(x) \notin K$. Next, recall that for any $v \in H \backslash K$, the defining inequality for $P v$ yields $\langle v-P v$, $P v-u\rangle \geqslant 0$ for all $u$ in $K$. Take $v=y+d \mu(x)$; to see that $P v=y$, we must show that for $u \in K$, we have $0 \leqslant\langle d \mu(x), y-u\rangle$, and this is immediate from $(*)$.

To prove (ii), we use the fact that $d \mu(P x)$ is the unique element of $H$ satisfying

$$
\langle d \mu(P x), u\rangle \leqslant 1 \quad(u \in K) \text { and }\langle d \mu(P x), P x\rangle=1 .
$$

On the other hand, the defining inequality shows that $\langle x-P x, P x-u\rangle \geqslant 0$ for all $u \in K$ and $\langle x-P x, P x\rangle$ is positive (since $0 \in$ int $K$ ), hence for all $u \in K$, $\langle x-P x, P x\rangle^{-1}\langle x-P x, u\rangle \leqslant 1$, with equality holding at $u=P x$. By uniqueness, $d \mu(P x)=\langle x-P x, P x\rangle^{-1}(x-P x)$.

As we will see below, except in some trivial instances $P_{K}$ is never differentiable (even Gateaux) at a boundary point of $K$. Our choice of definition of the Gateaux derivative, of course, requires that the differential be a linear operator; if one is willing to settle for the existence of a directional derivative in each direction, then this exists at every boundary point, for an arbitrary closed convex set $K$. This fact has been proved (independently) by Zarantonello [10] and Haraux [3] and generalized by Mignot [6]. We will describe this result, use it to characterize Gateaux smoothness of a convex body, and then prove an analogous characterization of Fréchet smoothness. 
Definition. If $K$ is a closed convex set and $x \in K$ we define the support cone $S_{K}(x)$ (or simply $S(x)$ ) of $K$ at $x$ to be the closure of the set

$$
\cap\{\lambda(K-x): \lambda \geqslant 0\} \text {. }
$$

This is a closed convex cone with vertex at the origin which "supports" $K$ in the sense that $K \subseteq x+S_{K}(x)$.

Zarantonello [10, p. 300] (see also Haraux [3]) has proved the following lemma. It has been extended to spaces with nonsymmetric inner product by Mignot [6].

3.2 Lemma (Zarantonello [10]). For any $x \in K$,

$$
P_{K}(x+t h)=x+t P_{S(x)} h+o(t), \quad t>0, h \in H .
$$

Thus, at each point $x$ of $K$, the map $P_{K}$ has a directional derivative in every direction $h$, given by $P_{S(x)} h$. Since the metric projection $P_{S(x)}$ will be linear only in the very special case when $S(x)$ is a linear subspace, it is clear that $d P(x)$ does not generally exist (in our sense) at points $x$ of $K$. In particular, if $K \neq H$ has nonempty interior and $x \in \partial K$, then $S_{K}(x)$ will not be a subspace, so $d P(x)$ will not exist. A "partial derivative" will sometimes exist, however.

Definition. Suppose $x \in H$ and let $M$ be a linear subspace of $H$. We say that $P$ has a partial Gateaux [resp. Fréchet] derivative in $M$ at $x$ provided there exists a bounded linear map $L$ of $M$ into itself (called the partial derivative) such that

$$
P(x+t h)=P x+t L h+o(t) \quad(h \in M),
$$

resp.

$$
P(x+h)=P x+L h+o(h) \quad(h \in M) .
$$

The next two results show that for $K$ with nonempty interior, the Minkowski functional for $K$ is Gateaux (resp. Fréchet) differentiable at a boundary point $x$ of $K$ if and only if the identity operator in $T[x]$ (the hyperplane through the origin parallel to the support hyperplane to $K$ at $x$ ) is the Gateaux (resp. Fréchet) partial derivative in $T[x]$ of $P_{K}$ at $x$. The first of these results is rather immediate from Zarantonello's lemma.

3.3 Proposition. Suppose that $0 \in$ int $K$, that $\mu$ is the Minkowski functional for $K$ and that $x \in \partial K$. If $d \mu(x)\left[\mu^{\prime}(x)\right]$ exists, then $P$ has the identity map in $T[x]=\{h$ : $\langle d \mu(x), h\rangle=0\}$ as its partial Gateaux [Fréchet] derivative in $T[x]$.

Proof. If $d \mu(x)$ exists, then $S=S_{K}(x)$ is the half-space $\{h:\langle h, d \mu(x)\rangle \leqslant 0\}$ bounded by $T[x]$. The restriction to $T[x]$ of the projection $P_{S}$ is therefore the identity map on $T[x]$ and Lemma 3.2 gives the desired conclusion. Suppose, next, that $\mu^{\prime}(x)$ exists; then for $h \in T[x]$ we have

$$
\mu(x+h)=\mu(x)+\left\langle\mu^{\prime}(x), h\right\rangle+o(h)=1+o(h) .
$$

Since $\left\langle\mu^{\prime}(x), u\right\rangle \leqslant \mu(u)$ for all $u$ and $\left\langle\mu^{\prime}(x), x\right\rangle=1$, we have $1=\left\langle\mu^{\prime}(x), x+h\right\rangle \leqslant$ $\mu(x+h)$ whenever $h \in T[x]$. Also, $\mu(x+h)^{-1}(x+h) \in K$ for $h$ near 0 and therefore

$$
\begin{aligned}
\|(x+h)-P(x+h)\| & \leqslant\left\|(x+h)-\mu(x+h)^{-1}(x+h)\right\| \\
& =\mu(x+h)^{-1}[\mu(x+h)-1]\|x+h\| .
\end{aligned}
$$


Since $\mu(x+h)^{-1}\|x+h\|$ is continuous in $h$ at $h=0$ we conclude that $P(x+h)=$ $x+h+o(h)(h \in T[x])$, which is precisely what we wanted to prove.

The converse to this result is slightly awkward to formulate; since we want to conclude that $d \mu(x)$ exists, we do not have it available to define the "tangent space" $T[x]$.

3.4 Proposition. Suppose that $0 \in$ int $K$, that $\mu$ is the Minkowski functional for $K$ and that $x \in \partial K$. Let $w \neq 0$ be any element of $H$ such that $P(x+w)=x$ and suppose that the identity map for the hyperplane

$$
H[x+w]=\{h \in H:\langle h, w\rangle=0\}
$$

is the partial Gateaux [Fréchet] derivative at $x$ for $P$ in $H[x+w]$. Then $\mu$ is Gateaux [Fréchet] differentiable at $x$.

Proof. By the defining inequality for $P(x+w)$ we have

$$
0<\sup \{\langle u, w\rangle: u \in K\}=\langle x, w\rangle \text {. }
$$

Since $P(x+r w)=P x=x$ for any $r>0$ we can assume that this supremum equals 1. From the definition and positive homogeneity of $\mu$ we conclude that

$$
\langle u, w\rangle \leqslant \mu(u) \quad(u \in H) \text { and }\langle x, w\rangle=1=\mu(x) .
$$

To prove Gateaux differentiability of $\mu$ at $x$ it suffices to show that $w$ is the unique element with these two properties. By hypothesis, for each $h \in H[x+w]$ we have

$$
P(x+t h)=x+t h+o(t) .
$$

Suppose that $v \in H$ is such that

$$
\langle u, v\rangle \leqslant \mu(u) \quad(u \in H) \text { and }\langle v, x\rangle=1 .
$$

Then for any $h$ satisfying $\langle h, w\rangle=0$ and any $t>0$ we have

$$
\begin{aligned}
1+t\langle h, v\rangle & =\langle x+t h, v\rangle \leqslant \mu(x+t h)=\mu[P(x+t h)+o(t)] \\
& \leqslant \mu[P(x+t h)]+\mu(o(t)) \leqslant 1+\mu(o(t))
\end{aligned}
$$

so that

$$
\langle h, v\rangle \leqslant t^{-1} \mu(o(t))=\mu\left(t^{-1} o(t)\right) .
$$

Thus, $\langle h, v\rangle \leqslant 0$; the same conclusion applies to $-h$ so $\langle h, v\rangle=0$ and hence $v$ is a scalar multiple of $w$. But $\langle x, v\rangle=1=\langle x, w\rangle$, so $v=w$ and hence $d \mu(x)$ exists (and equals $w$ ). To prove the assertion concerning Fréchet differentiability we first prove that, under the stated hypothesis that

we have

$$
P(x+h)=x+h+o(h), \quad h \in H[x+w],
$$

$$
\mu(x+h)=1+o(h), \quad h \in H[x+w] .
$$

Indeed, if $h \in H[x+w]$, then from (*) it follows that $1=\langle x+h, w\rangle \leqslant \mu(x+h)$ so $x+h$ is not in the interior of $K$ and therefore $P(x+h) \in \partial K$, that is, $\mu[P(x+h)]=1$. Since $\mu$ is continuous and homogeneous there exists $M>0$ such that $\mu(u) \leqslant M\|u\|$ for all $u \in H$. Thus, if $h \in H[x+w]$, then

$$
\begin{aligned}
0 & \leqslant \mu(x+h)-1=\mu(x+h)-\mu(P(x+h)) \\
& \leqslant \mu[x+h-P(x+h)] \leqslant M\|x+h-P(x+h)\|
\end{aligned}
$$


and this last term is $o(h)$. We will use this fact to show that $\mu^{\prime}(x)$ exists and equals $w$, that is,

$$
\mu(x+y)=1+\langle w, y\rangle+o(y), \quad y \in H .
$$

First note that we can always write

$$
y=h+\langle w, y\rangle x, \quad \text { where } h=y-\langle w, y\rangle x \in H[x+w] .
$$

If $\|y\|$ is sufficiently small, then $1+\langle w, y\rangle \geqslant 2^{-1}$, so assuming this we have

$$
\begin{aligned}
0 & \leqslant \mu(x+y)-\langle w, x+y\rangle=\mu(x+h+\langle w, y\rangle x)-1-\langle w, y\rangle \\
& =(1+\langle w, y\rangle)\left[\mu\left(x+(1+\langle w, y\rangle)^{-1} h\right)-1\right] .
\end{aligned}
$$

By what we proved above, this is $(1+\langle w, y\rangle) o\left((1+\langle w, y\rangle)^{-1} h\right)$. Since $|\langle w, y\rangle| \leqslant$ $\|w\|\|y\|$ and $\|h\| \leqslant(1+\|w\| \cdot\|x\|)\|y\|$, this last term is $o(y)$ and the proof is complete.

We have the following simple but useful proposition, which shows that surjectivity of the derivative of $P$ for a point of $x$ outside of $K$ implies the existence of a partial derivative of $P$ at $P x$. We need not assume that $K$ has an interior point.

3.5 Proposition. Suppose that $K$ is closed and convex and that $x \in H \backslash K$. If $d P(x)$ $\left[P^{\prime}(x)\right]$ exists and maps $H[x]$ onto itself, then for points $h$ in $H[x]$ we have $P(P x+t h)=P x+t h+o(t)[P(P x+h)=P x+h+o(h)]$, that is, the partial derivative of $P$ in $H[x]$ exists at $P x$ and equals the identity map in $H[x]$.

Proof. We will carry out the proof for the Fréchet derivative; the proof for the Gateaux derivative is similar. Let $A$ denote the restriction of $P^{\prime}(x)$ to the subspace $H[x]$; by hypothesis, $A$ is onto, hence it is invertible. We want to show that for $h \in H[x]$,

$$
P(P x+h)=P x+P^{\prime}(x) A^{-1} h+o(h),
$$

which is equivalent, once we write $u=A^{-1} h$ and note that $o\left(A^{-1} h\right)=o(h)$, to showing that for $u \in H[x]$

$$
P(P x+A u)=P x+P^{\prime}(x) u+o(u) .
$$

By hypothesis,

$$
P(x+u)=P x+P^{\prime}(x) u+o(u)
$$

since $P$ is nonexpansive, this implies that

$$
P(P(x+u))=P\left(P x+P^{\prime}(x) u\right)+o(u),
$$

that is

$$
P(x+u)=P(P x+A u)+o(u) .
$$

From (2) the left side equals $P x+P^{\prime}(x) u+o(u)$ which gives us (1) and completes the proof.

3.6 Corollary. Suppose that $0 \in$ int $K$ and that $x \in H \backslash K$. If $d P(x)\left[P^{\prime}(x)\right]$ exists and maps $H[x]$ onto itself, then $d \mu(P x)\left[\mu^{\prime}(P x)\right]$ exists and equals

$$
\langle P x, x-P x\rangle^{-1}(x-P x) \text {. }
$$


Proof. In the hypotheses to Proposition 3.4 we can take the boundary point to be $P x$ and $w=x-P x$, so that $H[x+w]=H[x]$. Proposition 3.5 shows that the remaining hypotheses are satisfied and we can therefore conclude that $d \mu(P x)$ $\left[\mu^{\prime}(P x)\right]$ exists and is given by $w$, provided the latter is normalized to have its supremum on $K$ (which is necessarily attained at $P x$ ) equal to 1 . Thus, either derivative has the indicated form.

The example in $\$ 5$ will show that the converse to this corollary is not valid.

The next result is analogous to Proposition 3.5, getting a weaker conclusion from a slightly weaker hypothesis.

3.7 Proposition. Suppose that $K$ is closed and convex and that $x \in H \backslash K$. If $d P(x)$ exists and is one-one in $H[x]$, then $H[x]+P x$ is the unique hyperplane supporting $K$ at Px.

Proof. If there existed another support hyperplane to $K$ at $P x$ we could choose $y \in H \backslash K$ such that $P y=P x$ and $y-P y \notin R(x-P x)$. The points of the segment $[x, y]$ would be mapped onto $P x$ by $P$ so for $0<t<1$, we would have

$$
P(x+t(y-x))=P x,
$$

which implies that $d P(x)(y-x)=0$. Consequently, if $P_{x}$ denotes the orthogonal projection of $H$ onto $H[x]$, we would have $d P(x)\left[P_{x}(y-x)\right]=d P(x)(y-x)=0$ (by Proposition 2.3). Since $d P(x)$ is assumed to be one-one on $H[x]$, this implies that $P_{x}(y-x)=0$. On the other hand, $P_{x}(y-x)=P_{x}(y-P x-(x-P x))=$ $P_{x}(y-P x) \neq 0$; otherwise, $y-P x \in H[x]^{\perp}=R(x-P x)$. This contradiction completes the proof.

3.8 Corollary. If $0 \in$ int $K$ and $d P(x)$ exists and is one-one in $H[x]$ for each $x \in H \backslash K$, then the Minkowski functional for $K$ is Gateaux differentiable wherever it is nonzero.

We next characterize $C^{2}$ (and higher) smoothness of $\partial K$; half of this characterization has been proved by Holmes [4].

3.9 THEOREM (Holmes). If $K$ has a $C^{k}$ boundary (where $k \geqslant 2$ ), then $P_{K}$ is $C^{k-1}$ in $H \backslash K$ and $P^{\prime}(x)$ is invertible in $H[x]$, for each $x \in H \backslash K$.

(The invertibility assertion does not appear in Holmes' statement of his theorem, but it is obvious from the formula he obtains for $P_{K}^{\prime}(x)$.)

3.10 TheOREM. Suppose that int $K \neq \varnothing$ and that for some $k \geqslant 1$ the map $P_{K}$ is $C^{k}$ in $H \backslash K$, with $P^{\prime}(x)$ invertible in $H[x]$ for each $x \in H \backslash K$; then the boundary of $K$ is $C^{k+1}$.

Proof. Without loss of generality we assume $0 \in$ int $K$ and we let $\mu$ denote the Minkowski functional for $K$. Fix a boundary point of $K$. We can assume that it is of the form $P x$ where $x \in H \backslash K$ and $\langle P x, x-P x\rangle=1$. By Proposition 3.5, the existence and invertibility of $P^{\prime}(x)$ imply that $\mu^{\prime}(P x)$ exists and is given by $\langle P x, x-P x\rangle^{-1}(x-P x)$. Consider the map $F: H \rightarrow H$ defined by

$$
F u=\langle u, x-P x\rangle P u, \quad u \in H .
$$


It is clear that $F$ is $C^{k}$ in $H \backslash K$ and its derivative at $u \in H \backslash K$ is easily calculated to be

$$
F^{\prime} u=\langle I, x-P x\rangle P u+\langle u, x-P x\rangle P^{\prime} u .
$$

We will verify below that $F^{\prime} x$ is an invertible operator in $H$. Suppose that this has been done; we can then apply the inverse function theorem to obtain open neighborhoods $U$ of $x$ and $V$ of $F x$ and a $C^{k}$ function $G: V \rightarrow U$ such that for $y \in V$ there exists $u \in U$ satisfying $y=F u$ and $G y=u$. From the inequalities $0<\|x-P x\|^{2}=\langle x, x-P x\rangle-\langle P x, x-P x\rangle\langle\langle x, x-P x\rangle$ it follows that $0<$ $\langle u, x-P x\rangle$ for all $u$ in some neighborhood of $x$, so there is no loss of generality in assuming that this holds in $U$. This implies that $F$ is a positive scalar multiple of $P$ in $U$ and hence by Proposition 3.1(iii), $\mu^{\prime}(P u)=\mu^{\prime}(F u)$ for $u$ in $U$. Consequently, if $y \in V$, so that $y=F u$ for some $u \in U$, then

$$
\begin{aligned}
\mu^{\prime}(y) & =\mu^{\prime}(F u)=\mu^{\prime}(P u)=\langle u-P u, P u\rangle^{-1}(u-P u) \\
& =\langle G y-P G y, P G y\rangle^{-1}(G y-P G y) .
\end{aligned}
$$

Since $G$ is $C^{k}$ this implies that $\mu^{\prime}$ is $C^{k}$ hence $\mu$ is $C^{k+1}$ in $V$. The latter is a neighborhood of $F x=\langle x, x-P x\rangle P x$ so by positive homogeneity of $\mu$, it is $C^{k+1}$ in a neighborhood of $P x$. This being true for each $x$ in $H \backslash K$, we get the desired conclusion.

It remains, of course, to prove that $F^{\prime} x$ is invertible; we will show that it is one-one and onto, then apply the open mapping theorem. Suppose, first, that $0=F^{\prime}(x) h=\langle h, x-P x\rangle P x+\langle x, x-P x\rangle P^{\prime}(x) h$. Taking the inner product of both sides with $x-P x$ yields $0=\langle h, x-P x\rangle\langle P x, x-P x\rangle$, so $\langle h, x-P x\rangle=0$ and hence $P^{\prime}(x) h=0$. Recall (from Proposition 2.3) that $P^{\prime}(x)=P^{\prime}(x)^{\circ} \circ P_{x}$, where $P_{x}$ is the orthogonal projection onto $H[x]$. Since (by hypothesis) $P^{\prime}(x)$ is one-one in $H[x]$, we conclude that $P_{x} h=0$ and hence $h=r(x-P x)$ for some $r \in R$. But then $0=\langle h, x-P x\rangle=r\langle x-P x, x-P x\rangle$, so $r=0$ and therefore $h=0$, which shows that $F^{\prime} x$ is one-one. To show that $F^{\prime} x$ is onto, suppose that $z \in H$. The orthogonal decomposition $H=H[x] \oplus R(x-P x)$ allows us to write

$$
z=P_{x} z+\|x-P x\|^{-2}\langle z, x-P x\rangle(x-P x) .
$$

Using the hypothesis that $P^{\prime} x$ is invertible in $H[x]$, let

$$
h=\langle x, x-P x\rangle^{-1}\left(P^{\prime} x\right)^{-1} P_{x}[z-\langle z, x-P x\rangle P x]+z-P_{x} z .
$$

Then from the formula for $F^{\prime} x$ we have

$$
\begin{aligned}
F^{\prime}(x) h & =\langle h, x-P x\rangle P x+\langle x, x-P x\rangle P^{\prime}(x) h \\
& =\langle z, x-P x\rangle P x+P_{x}[z-\langle z, x-P x\rangle P x] \\
& =\langle z, x-P x\rangle\left(P x-P_{x} P x\right)+P_{x} z .
\end{aligned}
$$

Now, $P x=P_{x} P x+\|x-P x\|^{-2}(x-P x)($ since $\langle P x, x-P x\rangle=1)$ and therefore

$$
F^{\prime}(x) h=\|x-P x\|^{-2}\langle z, x-P x\rangle(x-P x)+P_{x} z=z,
$$

so $F^{\prime} x$ is onto and the proof is complete.

One might hope that it is still possible to show the existence of $P^{\prime}$ in $H \backslash K$ (but not necessarily its invertibility in the appropriate tangent space) without assuming 
that $\mu$ is $C^{2}$. The example in $\S 5$ destroys this hope in a decisive way: $P^{\prime} x$ can fail to exist at each $x \in H \backslash K$ even though $\mu$ is $C^{1}$ with a locally Lipschitzian Fréchet derivative.

In the opposite direction, even in $R^{2}$ it is possible for $P$ to be $C^{1}$ in $H \backslash K$ while $\mu$ fails to be everywhere Gateaux differentiable; this will be shown in the next section.

Finally, we note that it is possible to prove a "point" version of Holmes' theorem: If $\mu$ is $C^{1}$ and if, for some $x \in H \backslash K$, the second derivative $\mu^{\prime \prime}(P x)$ exists, then $P^{\prime} x$ exists (and is invertible in $H[x]$ ). Our proof of this result is somewhat lengthy.

4. Metric projections of polar convex bodies. Suppose that $K$ is bounded, closed and convex and that $0 \in$ int $K$. Defining, as usual,

$$
K^{0}=\{y \in H:\langle x, y\rangle \leqslant 1 \text { for all } x \in K\},
$$

we know that $K^{0}$ is also bounded, closed and convex with the origin in its interior; moreover $K^{00}=K$. In what follows we will write $P$ and $P_{0}$ for $P_{K}$ and $P_{K^{0}}$ respectively; our task is to relate differentiability properties of $P_{0}$ to those of $P$.

Suppose that $x \in H \backslash K$; since $x-P x$ (considered as a linear functional) attains its supremum on $K$ at $P x$ and since $0 \in$ int $K$, we have

$$
\langle x-P x, P x\rangle>0 \text {. }
$$

Let $Q x=\langle x-P x, P x\rangle^{-1}(x-P x)$; then the supremum of $Q x$ on $K$ equals 1 , and hence $Q x \in K^{0}$ and $\langle Q x, P x\rangle=1$. Furthermore, from the definition of $K^{0}, P x=$ $(Q x+P x)-Q x$ attains its supremum (equal to 1$)$ on $K^{0}$ at $Q x$, which implies that $Q x+P x \in H \backslash K^{0} \quad$ (since $\langle P x, Q x+P x\rangle=1+\|P x\|^{2}>1=\langle P x, Q x\rangle$ ) and $P_{0}(Q x+P x)=Q x$. If $\lambda>0$, then $Q x+\lambda P x$ lies on the ray from $Q x$ through $Q x+P x$, so

$$
P_{0}(Q x+\lambda P x)=Q x \quad \text { for all } x \in H \backslash K, \lambda>0 .
$$

We will exploit this relationship to compute the derivative of $P_{0}$ in terms of $P$. We first do this for points of a special form; Proposition 2.5 then does it for arbitrary points.

4.1 Proposition. Suppose that $x \in H \backslash K$, that $x=P x+Q x$, and that $d P(x)$ $\left[P^{\prime}(x)\right]$ exists. Then $x \in H \backslash K^{0}$ and $d P_{0}(x)\left[P_{0}^{\prime}(x)\right]$ exists.

Proof. As we showed above, $x=P x+Q x \in H \backslash K^{0}$; moreover, $\langle x-P x, P x\rangle$ $=\langle Q x, P x\rangle=1$. We will carry out the details of the proof for $d P_{0}(x)$; it will be clear that a parallel argument yields the result for $P_{0}^{\prime}(x)$. It is obvious that if $d P(x)$ $\left[P^{\prime}(x)\right]$ exists, then so does $d Q(x)\left[Q^{\prime}(x)\right]$. To see what form the derivative of $P_{0}$ must have, consider $y \in H \backslash K$ and write $\lambda=\langle y-P y, P y\rangle^{-1}$. With $y$ in place of $x$ and this choice of $\lambda>0$ in (1) we obtain the identity

$$
P_{0}\left(\langle y-P y, P y\rangle^{-1} y\right)=Q y .
$$

If both $P$ and $P_{0}$ were Fréchet differentiable, say, then the chain rule would yield

$$
P_{0}^{\prime}\left(\langle y-P y, P y\rangle^{-1} y\right) \circ A^{\prime}(y)=Q^{\prime}(y)
$$


where $A y=\langle y-P y, P y\rangle^{-1} y$. At the special point $y=P x+Q x=x$ we have $\langle x-P x, P x\rangle=1$ so this yields

$$
P_{0}^{\prime}(x)=Q^{\prime}(x) \circ A^{\prime}(x)^{-1},
$$

provided $A^{\prime}(x)$ is invertible. In fact, if $d P(x)$ exists, then $d A(x)$ is invertible and

$$
d P_{0}(x)=d Q(x) \circ d A(x)^{-1} .
$$

To see the first assertion, note that

$$
d A(x)=I-\langle(I-d P(x))(\cdot), P x\rangle x .
$$

(Here we have used $\langle x-P x, d P(x)\rangle=0$ and $\langle x-P x, P x\rangle=1$.) Straightforward calculations show that $d A(x)$ is one-one and onto, with (bounded) inverse

$$
d A(x)^{-1}=I+(1-\langle x-d P(x) x, P x\rangle)^{-1}\langle(I-d P(x))(\cdot), P x\rangle x,
$$

provided $\langle x-d P(x) x, P x\rangle \neq 1$. To see that the latter always holds, suppose $1=$ $\langle x-d P(x) x, P x\rangle=\langle Q x+P x-d P(x) x, P x\rangle=1+\|P x\|^{2}-\langle d P(x) x, P x\rangle$. Since (Proposition 2.3) $d P(x)(x-P x)=0$, we have $\langle d P(x) P x, P x\rangle=\|P x\|^{2}$. Since $\|d P(x)\| \leqslant 1$ we have $\|d P(x) P x\| \leqslant\|P x\|$ so it follows that $d P(x) P x=P x$. But then $0=\langle d P(x) P x, x-P x\rangle=\langle P x, x-P x\rangle=\langle P x, Q x\rangle=1$, a striking contradiction which shows that $d A(x)$ is invertible. It remains, then, to prove (2). To this end, fix $v \in H$; we want

$$
P_{0}(x+t v)-P_{0}(x)=t d Q(x) d A(x)^{-1} v+o(t),
$$

where $o(t) / t \rightarrow 0$ as $t \rightarrow 0$. Writing $u=d A(x)^{-1} v$ this is equivalent to

$$
P_{0}(x+t d A(x) u)-P_{0}(x)=t d Q(x) u+o(t) .
$$

The derivative $d Q(x)$ is readily computed explicitly; it is given by

$$
d Q(x) u=u-d P(x) u-\langle u-d P(x) u, P x\rangle(x-P x) .
$$

By using $x=P x+Q x$ and writing $\alpha$ for $\langle u-d P(x) u, P x\rangle$, we can now apply formula (3) to obtain

$$
x+t d A(x) u=(1-t \alpha) P x+Q x+t[d P(x)+d Q(x)] u .
$$

On the other hand, $P(x+t u)=P x+t d P(x) u+o(t)$ and $Q(x+t u)=Q x+$ $t d Q(x) u+o(t)$, so (using the fact that $t^{2} \alpha d P(x) u$ is $o(t)$ )

$$
x+t d A(x) u=(1-t \alpha) P(x+t u)+Q(x+t u)+o(t) .
$$

Since $P_{0}$ is nonexpansive, this implies that

$$
\begin{aligned}
P_{0}(x+t d A(x) u) & =P_{0}[(1-t \alpha) P(x+t u)+Q(x+t u)]+o(t) \\
& =Q(x+t u)+o(t),
\end{aligned}
$$

by virtue of $(1)$ and the fact that $1-t \alpha>0$ for all sufficiently small $|t|$. Thus,

$$
P_{0}(x+t d A(x) u)-P_{0}(x)=Q(x+t u)-Q(x)+o(t)=d Q(x) u+o(t),
$$

which proves (4) and completes the proof of the proposition.

4.2 Corollary. If $d P\left[P^{\prime}\right]$ exists in $H \backslash K$, then $d P_{0}\left[P_{0}^{\prime}\right]$ exists in $H \backslash K^{0}$. If $P$ is $C^{1}$ in $H \backslash K$, then $P_{0}$ is $C^{1}$ in $H \backslash K^{0}$. 
Proof. To prove the first assertion, it suffices to show that any point $y \in H \backslash K^{0}$ lies on a ray of the form $\{Q x+\lambda P x: \lambda>0\}$ for some $x \in H \backslash K$ satisfying $x=P x+Q x$. Indeed, the last proposition then guarantees that $P_{0}$ is differentiable at $x$ and, since $x=P x+Q x$, equation (1) implies that $Q x=P_{0} x$ and $P x=x-P_{0} x$, so that $Q x+\lambda P x=\lambda x+(1-\lambda) P_{0} x$ whenever $\lambda>0$ and therefore Proposition 2.5 shows that $P_{0}$ is differentiable at $y$. Given $y \in H \backslash K^{0}$, then, we choose suitable $x$ and $\lambda$ as follows. First, we define $Q_{0}$ in terms of $P_{0}$ as we did $Q$ :

$$
Q_{0} u=\left\langle u-P_{0} u, P_{0} u\right\rangle^{-1}\left(u-P_{0} u\right) \quad\left(u \in H \backslash K^{0}\right) .
$$

We next let $x=P_{0} y+Q_{0} y$. If we reverse the roles of $K$ and $K^{0}$ in our initial discussion, we see immediately that $x \in H \backslash K$ and that

$$
P x=P\left(P_{0} y+Q_{0} y\right)=Q_{0} y .
$$

Moreover, using this identity we obtain $\langle x-P x, P x\rangle=\left\langle x-Q_{0} y, Q_{0} y\right\rangle=$ $\left\langle P_{0} y, Q_{0} y\right\rangle=1$, so that $Q x=x-P x=x-Q_{0} y=P_{0} y$. Finally, we can write (by definition of $Q_{0} y$ )

$$
y=\left\langle y-P_{0} y, P_{0} y\right\rangle Q_{0} y+P_{0} y=\lambda P x+Q x,
$$

with $\lambda=\left\langle y-P_{0} y, P_{0} y\right\rangle>0$.

To prove the second assertion we combine the formulas we have already obtained. First, Proposition 2.5 shows that when $y=\lambda x+(1-\lambda) P_{0} x$, then

$$
P_{0}(y)=P_{0}^{\prime}(x) \circ\left[\lambda I+(1-\lambda) P_{0}^{\prime}(x)\right]^{-1} .
$$

Next, the proof of Proposition 4.1 shows that

$$
P_{0}^{\prime}(x)=Q^{\prime}(x) \circ A^{\prime}(x)^{-1},
$$

where $A u=\langle u-P u, P u\rangle^{-1} u$. It is clear that when $P$ is $C^{1}$, then $Q^{\prime}(x)$ and $A^{\prime}(x)^{-1}$ are continuous (for $x \in H \backslash K$ ). Finally, from what we have just proved, both $x$ and $\lambda$ are continuous functions of $y$; by appropriate substitutions we see that $P_{0}^{\prime}(y)$ is continuous in $y$.

This corollary leads to easy examples showing that one cannot deduce much about the smoothness of a convex body merely from the differentiability of its metric projection.

4.3 EXAMPLE. There exists a centrally symmetric convex body $K^{0}$ in $\mathbf{R}^{2}$ whose metric projection is $C^{1}$ in $\mathbf{R}^{2} \backslash K^{0}$ but $K^{0}$ does not have a Gateaux differentiable Minkowski functional.

Proof. It is easy to construct $C^{2}$ smooth centrally symmetric convex bodies $K$ in $\mathbf{R}^{2}$ whose boundaries contain straight line segments. The polar $K^{0}$ of such a set will have "corners", hence will not be (Gateaux) smooth. By Holmes' theorem (Theorem 3.9), the metric projection for $K$ will be $C^{1}$ in $\mathbf{R}^{2} \backslash K$, hence by Corollary 4.2, the projection for $K^{0}$ will be $C^{1}$ in $\mathbf{R}^{2} \backslash K^{0}$.

One can also write down explicit formulas for such examples; for instance, the graph of the convex function $f(x)=x^{4 / 3}+|x|$ can be considered to define a convex body $K$ in $\mathbf{R}^{2}$ which clearly fails to be differentiable at 0 . A laborious verification shows that the metric projection of $\mathbf{R}^{2}$ onto $K$ is $C^{1}$. 
5. An example. Throughout most of this section we will work in the separable Hilbert space $L^{2}[0,1]$ (Lebesgue measure).

Notation. Let $B_{\infty}=\left\{f \in L^{2}:|f| \leqslant 1\right.$ a.e. $\}, B_{2}=\left\{f \in L^{2}:\|f\| \leqslant 1\right\}$, and $B=B_{\infty}$ $+B_{2}$.

It is clear that $B_{\infty}$ is a bounded closed convex and symmetric set and that (since $B_{2}$, say, is weakly compact) the set $B$ is closed, as well as being bounded, symmetric, and convex, with the origin in its interior.

More notation. Let $P_{\infty}$ and $P$ denote the metric projections of $L^{2}$ onto $B_{\infty}$ and $B$, respectively, and let \|\|$\cdot \|$ denote the (equivalent) norm on $L^{2}$ defined by the Minkowski functional for $B$. The $L^{1}$ norm on $L^{2}$ is denoted by $\|\cdot\|_{1}$.

5.1 Proposition. (1) The metric projection $P$ onto $B$ is nowhere Fréchet differentiable, despite the fact that

(2) The norm $\||\cdot \cdot \||$ defined by $B$ is Fréchet differentiable at every nonzero point and its differential map $D: L^{2} \backslash\{0\} \rightarrow L^{2}$ is locally Lipschitzian.

(3) $P$ is Gateaux differentiable at $f \in L^{2}$ if and only if $\{t:|f(t)|=1\}$ has measure zero.

The proofs of these three assertions will eventually emerge from a (finite) sequence of preliminary results.

1. For $f \in L^{2}$ we have

$$
P_{\infty} f= \begin{cases}f & \text { on }\{t \in[0,1]:|f(t)| \leqslant 1\}, \\ \operatorname{sgn} f & \text { elsewhere. }\end{cases}
$$

Equivalently, if $G: R \rightarrow R$ is defined by

$$
G(t)= \begin{cases}t, & |t| \leqslant 1, \\ \operatorname{sgn} t, & |t|>1,\end{cases}
$$

then $P_{\infty} f=G \circ f$. It is clear that $P_{\infty} f \in B_{\infty}$ for each $f \in L^{2}$; to see that $G \circ f$ is the nearest point to $f$, suppose that $g \in B_{\infty}$, so that $|f|-1 \leqslant|f|-|g| \leqslant|f-g|$. We then have (letting $A=\{t:|f(t)|>1\}, B=[0,1] \backslash A$ )

$$
\begin{aligned}
\|f-g\|_{2}^{2} & \geqslant \int_{A}|f-g|^{2} \geqslant \int_{A}(|f|-1)^{2}=\int_{A} f^{2}-2 \int_{A}|f|+\int_{A} 1 \\
& =\int f^{2}-2 \int_{B} f^{2}-2 \int_{A}|f|+\int_{B} f^{2}+\int_{A} 1 \\
& =\int f^{2}-2 \int f G \circ f+\int(G \circ f)^{2}=\|f-G \circ f\|^{2},
\end{aligned}
$$

which was to be proved.

2. The projection $P_{\infty}$ is nowhere Fréchet differentiable. This is a consequence of Vainberg's result [9] that a map of the form $f \rightarrow G \circ f$ ( $G$ continuous) is Fréchet differentiable at some point if and only if $G$ is affine.

3. The Gateaux derivative $d P_{\infty}(f)$ exists at $f$ if and only if $\{t:|f(t)|=1\}$ has measure zero. 
This can be deduced from Vainberg's methods [9] or proved directly; the sufficiency portion uses the fact that for $r \neq \pm 1$, the derivative $G^{\prime}(r)$ exists, has modulus at most 1 and is continuous. One uses the mean value theorem for $G$ and dominated convergence to show that $d P_{\infty}(f) h=\left(G^{\prime} \circ f\right) h$.

The metric projection $P$ can be expressed in terms of $P_{\infty}$ by using the following general result.

5.2 Lemma. Let $E$ be a strictly convex space and let $K$ be a weakly compact convex subset of $E$. If $U$ denotes the closed unit ball of $E$, then $B=K+U$ is a closed convex set and its (set-valued) metric projection $P$ is given, for $x \in E \backslash B$, by

$$
P(x)=\left\{y+\frac{x-y}{\|x-y\|}: y \in P_{K}(x)\right\} .
$$

Proof. The weak compactness of $K$ guarantees that $B$ is weakly closed, hence closed. Our computations will be aided by the elementary observation that if $x \in E \backslash B$, then

$$
\inf \{\|x-v\|: v \in K\}=\inf \{\|x-v-u\|: v \in K, u \in U\}+1 .
$$

Suppose, now, that $x \in E \backslash B$ and that $y+z(y \in K, z \in U)$ is a (typical) element of $P x$. Then

$$
\begin{aligned}
\|x-y\|-1 & =\inf \{\|x-y-v\|: v \in U\} \\
& \geqslant\|x-y-z\| \geqslant\|x-y\|-\|z\| \geqslant\|x-y\|-1 .
\end{aligned}
$$

It follows that $\|z\|=1$ and $\|x-y-z\|+\|z\|=\|x-y\|$; by strict convexity, this leads to $z=\|x-y\|^{-1}(x-y)$. It remains to show that $y \in P_{K} x$. We have just shown that $\|x-y\|=\|x-y-z\|+1$, while the observation above shows that this equals $\inf \{\|x-v\|: v \in K\}$, hence $y \in P_{K} x$.

To prove the reverse inclusion, suppose that $y \in P_{K} x$, we want to show that $y+\|x-y\|^{-1}(x-y) \in P x$. But the observation above shows that

$$
\begin{aligned}
\left\|x-\left[y+\|x-y\|^{-1}(x-y)\right]\right\| & =\|x-y\|-1 \\
& =\inf \{\|x-v-u\|: v \in K, u \in U\},
\end{aligned}
$$

which completes the proof.

4. The metric projection $P$ fails to be Fréchet differentiable at each point of $L^{2} \backslash B$, while it is Gateaux differentiable at $f \in L^{2} \backslash B$ if and only if $\{t:|f(t)|=1\}$ has measure zero.

Proof. The previous lemma shows that for $f \in L^{2} \backslash B$,

$$
P f=P_{\infty} f+\left\|f-P_{\infty} f\right\|^{-1}\left(f-P_{\infty} f\right) .
$$

Moreover, the proof shows that $\left\|f-P_{\infty} f\right\|=1+\|f-P f\|$. Now, it is a general property of metric projections in Hilbert space that the real-valued distance function

$$
f \rightarrow\|f-P f\|
$$

is Fréchet differentiable in $L^{2} \backslash B$. (See, for instance, [4].) We can rewrite the equation given above to obtain, for $f \in L^{2} \backslash B$,

$$
P_{\infty} f=\|f-P f\|^{-1}[(1+\|f-P f\|) P f-f],
$$


so if $d P(f)$ [or $P^{\prime}(f)$ ] exists, then so does $d P_{\infty}(f)$ [or $P_{\infty}^{\prime}(f)$ ]; similarly, existence of $d P_{\infty}(f)$ implies the same for $d P(f)$ (using the first formula). The two assertions are now immediate from parts 2 and 3.

It remains to prove part (2) of Proposition 5.1 concerning the norm $\||\cdot|||$ induced by $B$. To this end we compute the dual norm to $\|\cdots \cdot\| \|$.

5. If $f \in L^{2}$, then

$$
\sup \left\{|\langle f, g+h\rangle|: g \in B_{\infty}, h \in B_{2}\right\}=\|f\|_{1}+\|f\|,
$$

and this supremum is attained (for $f \neq 0$ ) at $g+h$ for any $g \in B_{\infty}$ such that $g=\operatorname{sgn} f$ on $\{t: f(t) \neq 0\}$ and for $h=\|f\|^{-1} f$.

PRoof. If $g \in B_{\infty}$ and $h \in B_{2}$, then

$$
|\langle f, g+h\rangle| \leqslant \int|g f|+\int|h f| \leqslant \int|f|+\left(\int|f|^{2}\right)^{1 / 2}=\|f\|_{1}+\|f\| .
$$

On the other hand, if $f \neq 0$, then for the indicated choices of $g$ and $h$ it is clear that the inequalities above become equality.

Since the sum of any two norms is strictly convex whenever one of them is strictly convex the result above shows that the norm dual to $\|\cdot \cdots\|$ is strictly convex, hence III - || $\mid$ itself is smooth; that is, it is Gateaux differentiable at each nonzero point $u \in L^{2}$. We need to relate the differential at $u$ to $u$ itself.

6. If $\|u\|=1$, then the Gateaux differential $D u$ of $\|\cdot\|$ at $u$ is the unique nonzero element $f \in L^{2}$ satisfying: $\|f\|_{1}+\|f\|=1$ and $u=u_{1}+\|f\|^{-1} f$, where $u_{1}=\operatorname{sgn} f$ on $\{t: f(t) \neq 0\}$ and $\left|u_{1}\right| \leqslant 1$ elsewhere.

Proof. The differential $D u$ is the unique element $f$ of $L^{2}$ which has dual norm equal to 1 and which, as a functional on $L^{2}$, attains its supremum on $B$ at $u$; by part 5 , this means we can write $u$ in the indicated form.

7. If $\|u\|=1=\|v\|$, then $\|D u-D v\| \leqslant 2\|u-v\|$.

Proof. Let $f=D u, g=D v$ and apply part 6 to write $u=u_{1}+\|f\|^{-1} f, v=v_{1}$ $+\|g\|^{-1} g$, where $u_{1}, v_{1}$ have the indicated properties and

$$
\|f\|_{1}+\|f\|=1=\|g\|_{1}+\|g\| \text {. }
$$

By the usual expansion we have

$$
\begin{aligned}
\|u-v\|^{2}= & \left\|u_{1}-v_{1}\right\|^{2}+2 \int\left(u_{1}-v_{1}\right)\left(\|f\|^{-1} f-\|g\|^{-1} g\right) \\
& +\left\|\left(\|f\|^{-1} f-\|g\|^{-1} g\right)\right\| .
\end{aligned}
$$

The middle term is twice

$$
\begin{aligned}
\|f\|^{-1} \int\left(u_{1} f-v_{1} f\right) & +\|g\|^{-1} \int\left(v_{1} g-u_{1} g\right) \\
= & \|f\|^{-1} \int\left(|f|-v_{1} f\right)+\|g\|^{-1} \int\left(|g|-u_{1} g\right) \geqslant 0
\end{aligned}
$$

since $v_{1} f \leqslant|f|$ and $u_{1} g \leqslant|g|$. Thus,

$$
\|u-v\| \geqslant\left\|\left(\|f\|^{-1} f-\|g\|^{-1} g\right)\right\| .
$$


Now, by the triangle inequality

$$
\|f-g\| \leqslant\|f\| \cdot\left\|\left(\|f\|^{-1} f-\|g\|^{-1} g\right)\right\|+\left\|\left(\|f\| \cdot\|g\|^{-1} g-g\right)\right\| .
$$

The first summand is dominated by $\|u-v\|$ since $\|f\|<1$. The second term is (using the norm constraints on $f$ and $g$ )

$$
\begin{aligned}
& |\|f\|-\|g\||=\left|\|f\|\left(\|g\|_{1}+\|g\|\right)-\|g\|\left(\|f\|_{1}+\|f\|\right)\right| \\
& \quad=\left|\|f\| \cdot\|g\|_{1}-\|g\| \cdot\|f\|_{1}\right| \leqslant\|(\|f\| g-\|g\| f)\|_{1} \\
& \quad \leqslant\|(\|f\| g-\|g\| f)\|=\|f\| \cdot\|g\| \cdot\left\|\left(\|g\|^{-1} g-\|f\|^{-1} f\right)\right\| \leqslant\|u-v\|,
\end{aligned}
$$

which completes the proof of 7 .

To complete assertion (2) of the proposition we recall several facts. First, for any element $x \neq 0$ of a Banach space with Gateaux differentiable norm, the differential $D(x)$ of the norm at $x$ is a functional of norm one and $D(r x)=D(x)$ for any $r>0$. If it is true that there exists $M>0$ such that

$$
\|D(x)-D(y)\| \leqslant M\|x-y\|
$$

whenever $\|x\|=1=\|y\|$, then for arbitrary nonzero $x$ and $y$ we have

$$
\begin{aligned}
\|D(x)-D(y)\| & =\left\|D\left(\|x\|^{-1} x\right)-D\left(\|y\|^{-1} y\right)\right\| \\
& \leqslant M\left\|\left(\|x\|^{-1} x-\|y\|^{-1} y\right)\right\| .
\end{aligned}
$$

Now,

$$
\begin{aligned}
\|\| x\left\|^{-1} x-\right\| y\left\|^{-1} y\right\| & =\|x\|^{-1}\left\|\left(x-\|x\| \cdot\|y\|^{-1} y\right)\right\| \\
& \leqslant\|x\|^{-1}\left[\|x-y\|+\|y-\| x\|\cdot\| y\left\|^{-1} y\right\|\right] \\
& =\|x\|^{-1}\left[\|x-y\|+\left|1-\|x\| \cdot\|y\|^{-1}\right|\|y\|\right] \\
& \leqslant\|x\|^{-1} 2\|x-y\| .
\end{aligned}
$$

It follows that if $D$ is Lipschitzian on the unit sphere, then it is Lipschitzian in the complement of any neighborhood of 0 , and locally Lipschitzian in $E \backslash\{0\}$. The fact that the norm $\|\cdot \cdot\|||$ is Fréchet differentiable in this same set is a consequence of the general fact referred to in $\$ 3$, namely that a norm to norm continuous Gateaux differential of a convex function is, in fact, the Fréchet differential.

We conclude with an example which has been mentioned previously by Mignot [6] (in the nonseparable case).

EXAMPLE. Let $\Gamma$ be a nonempty set and let $K$ be the closed convex positive cone in $l^{2}(\Gamma)$; that is,

$$
K=\{x=(x(\gamma)): x(\gamma) \geqslant 0 \text { for all } \gamma \in \Gamma\} .
$$

(i) For $x \in l^{2}(\Gamma)$, we have $P_{K}(x)=\left(x(\gamma)^{+}\right)$.

(ii) If $\Gamma$ is uncountable, then $P_{K}$ is nowhere Gateaux differentiable.

(iii) If $\Gamma$ is countably infinite, then $P_{K}$ is nowhere Fréchet differentiable and is Gateaux differentiable at $x=\left(x_{n}\right)$ if and only if $x_{n} \neq 0$ for all $n$.

Proof. Part (i) is easy to verify, and it is also easy to check that if $x(\gamma)=0$ for some $\gamma$, then the derivative of $P_{K}$ in the $\gamma$-coordinate direction fails to exist. This proves the only if part of (iii) and all of (ii). If $x_{n} \neq 0$ for all $n$, then one checks that 
$d P(x)=\left(\operatorname{sgn} x_{n}^{+}\right)$. Finally, if $x_{n} \neq 0$ for all $n$, with (say) $x_{n}>0$ for infinitely many $n$, let $\alpha_{n}=-2 x_{n}$ for such values of $n$ and then verify that

$$
\alpha_{n}^{-1}\left[P\left(x+\alpha_{n} \delta_{n}\right)-P x-\alpha_{n} d P(x) \delta_{n}\right] \nrightarrow 0,
$$

where $\delta_{n}$ is the $n$th coordinate vector in $l_{2}$. This shows that $P^{\prime}(x)$ does not exist and completes the proof.

\section{REFERENCES}

1. Edgar Asplund, Differentiability of the metric projection in finite-dimensional Euclidean space, Proc. Amer. Math. Soc. 38 (1973), 218-219.

2. J. Dieudonné, Foundations of modern analysis, Academic Press, New York and London, 1960.

3. A. Haraux, How to differentiate the projection on a convex set in Hilbert space. Some applications to variational inequalities, J. Math. Soc. Japan 29 (1977), 615-631.

4. Richard B. Holmes, Smoothness of certain metric projections on Hilbert space, Trans. Amer. Math. Soc. 184 (1973), 87-100.

5. J. Kruskal, Two convex counterexamples: a discontinuous envelope function and a non-differentiable nearest point mapping, Proc. Amer. Math. Soc. 23 (1969), 697-703.

6. F. Mignot, Contrôle dans les inéquations variationelles élliptiques, J. Funct. Anal. 22 (1976), 130-185.

7. R. R. Phelps, Gaussian null sets and differentiability of Lipschitz maps on Banach spaces, Pacific J. Math. 18 (1978), 523-531.

8. A. Wayne Roberts and Dale E. Varberg, Convex functions, Academic Press, New York and London, 1973.

9. M. M. Vainberg, Some questions of differential calculus in linear spaces, Uspehi Mat. Nauk (N.S.) 7(50), (1952), 55-102. (Russian)

10. E. H. Zarantonello, Projections on convex sets in Hilbert space and spectral theory, Contributions to Nonlinear Functional Analysis, Publ. No. 27, Math. Res. Center, Univ. Wisconsin, Academic Press, New York and London, 1971, pp. 237-424.

11. , L'algèbre des projecteurs coniques, Analyse Convexe et Ses Applications, Lecture Notes in Econom. and Math. Systems, vol. 102, Springer-Verlag, Berlin and New York, 1974, pp. 232-243.

Department of Mathematics, University of Washington, Seattle, Washington 98195 (Current address of R. R. Phelps)

Current address (Simon Fitzpatrick): Department of Mathematics, University of Illinois, Urbana, Illinois 61801 